\title{
Analytical device model for graphene bilayer field-effect transistors using weak nonlocality approximation
}

\author{
V. Ryzhii*1,4, M. Ryzhii ${ }^{1,4}$, A. Satou ${ }^{2,4}$, T. Otsuji ${ }^{2,4}$, and V. Mitin ${ }^{3}$ \\ ${ }^{1}$ Computational Nanoelectronics Laboratory, \\ University of Aizu, Aizu-Wakamatsu 965-8580, Japan \\ ${ }^{2}$ Research Institute for Electrical Communication, \\ Tohoku University, Sendai 980-8577, Japan \\ ${ }^{3}$ Department of Electrical Engineering, University at Buffalo, \\ State University of New York, NY 14260, USA \\ ${ }^{4}$ Japan Science and Technology Agency, \\ CREST, Tokyo 10\%-0075, Japan
}

\begin{abstract}
We develop an analytical device model for graphene bilayer field-effect transistors (GBL-FETs) with the back and top gates. The model is based on the Boltzmann equation for the electron transport and the Poisson equation in the weak nonlocality approximation for the potential in the GBL-FET channel. The potential distributions in the GBL-FET channel are found analytically. The source-drain current in GBL-FETs and their transconductance are expressed in terms of the geometrical parameters and applied voltages by analytical formulas in the most important limiting cases. These formulas explicitly account for the short-gate effect and the effect of drain-induced barrier lowering. The parameters characterizing the strength of these effects are derived. It is shown that the GBL-FET transconductance exhibits a pronounced maximum as a function of the top-gate voltage swing. The interplay of the short-gate effect and the electron collisions results in a nonmonotonic dependence of the transconductance on the top-gate length.
\end{abstract}

\section{INTRODUCTION}

Unique properties of graphene layers, graphene nanoribbon arrays and graphene bilayers [1-3] as well as graphene nanomeshs [4] make them promising for different nanoelectronic device applications. The gapless energy spectrum of graphene layers allows to use them in terahertz and midinfrared detectors and lasers [59]. However, the gapless energy spectrum of GLs is an obstacle for creating transistor digital circuits based on graphene field-effect transistors (G-FETs) due to relatively strong interband tunneling in the FET offstate [10, 11]. The reinstatement of the energy gap in graphene-based structures like graphene nanoribbons, graphene nanomeshs, and graphene bilayers appears to be unavoidable to fabricate FETs with a sufficiently large on/off ratio. Recently, the device dc and ac characteristics of graphene nanoribbon and graphene bilayer FETs (which are referred to as GNR-FETs and GBL-FETs, respectively) were assessed both numerically and analytically [12 19]. The device characteristics of GNR-FETs operating in near ballistic and drift-diffusion regimes can be calculated analogously with those of nanowire- and carbon nanotube-FETs (see, for instance 2022$]$ and references therein). The GBL-FET characteristics can, in principle, be found using the same approaches as those realized previously for more customary FETs with a twodimensional electron system in the channel 23 25, 27? 31]. However, some important features of GBL-FETs,

*Electronic mail: v-ryzhii@u-aizu.ac.jp in particular, the dependence of both the electron density and the energy gap in different sections of the GBL-FET channel on the gate and drain voltages should be considered [32-34], as well as the "short-gate" effect and the drain-induced barrier lowering [29].

In this paper, we use a substantially generalized version of the GBL-FET analytical device model [17, 18] to calculate the characteristics of GBL-FETs (the threshold voltages, current-voltage characteristics, and transconductance) in different regimes and analyze the possibility of a significant improvement of the ultimate performance of these FETs by shortening of the gate and decreasing of the gate layer thickness. The device model under consideration, which presents the GBL-FET characteristics in closed analytical form, allows a simple and clear evaluation of the ultimate performance of GBL-FETs and their comparison. The following effects are considered: (a) Dependences of the electron density and energy gap in different sections of the channel on the applied voltages and the inversion of the gated section charge; (b) Degeneracy of the electron system, particularly, in the source and drain sections of the channel; (c) The short-gate effect and the effect of drain-induced barrier lowering; (d) Electron scattering in the channel.

Our model is based on the Boltzmann kinetic equation for the electron system in the GBL-FET and the Poisson equation in the weak nonlocality approximation [35, 36]. The use of the latter allows us to find the potential distributions along the channel in the most interesting cases and obtain the GBL-FET characteristics analytically.

The paper is organized as follows. In Sec. II, the GBLFET device model under consideration is presented and the features of GBL-FET operation are discussed. In 


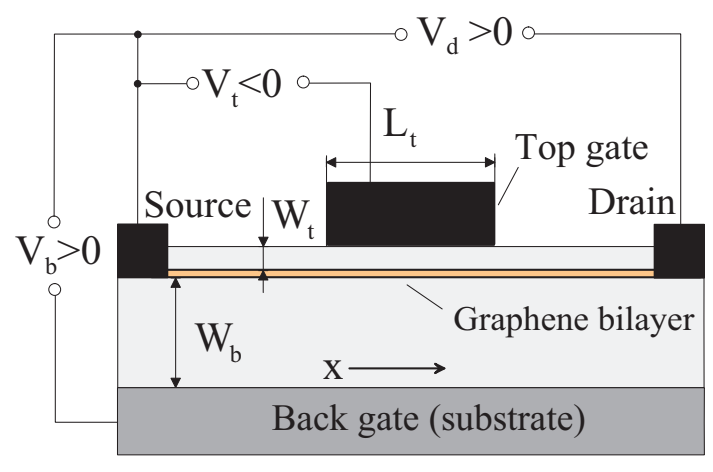

FIG. 1: Schematic view of the GBL-FET structure.

Sec. III, the main equations of the model are cited. The general formulas for the source-drain current and the GBL-FET transconductance simplified for the limiting cases (far below the threshold, near threshold, and at low top-gate voltages corresponding to the on-state) are also presented. In this section, the source-drain current and the GBL-FET transconductance are expressed in terms of the Fermi energy in the source and drain contacts and the height of the potential barrier in the channel. To find the barrier height, the Poisson equation is solved for different limiting cases in Sec. IV. The obtained potential distributions are used for the derivation of the explicit formulas for source-drain current and the transconductance as functions of the applied voltages and geometrical parameters. Section V deals with a brief discussion of some effects (role of the device geometry, electron scattering, charge inversion in the channel, and interband tunneling), which influence the GBL-FET characteristics. In Sec. VI, we draw the main conclusions. Some reference data related to the voltage dependences of the Fermi energy and the energy gap in different sections of the GBL-FET channel are singled out to the Appendix.

\section{DEVICE MODEL AND FEATURES OF OPERATION}

We consider a GBL-FET with the structure shown in Fig. 1. It is assumed that the back gate, which is positively biased by the pertinent voltage $V_{b}>0$, provides the formation of the electron channel in the GBL between the Ohmic source and drain contacts. A relatively short top gate serves to control the source-drain current by forming the potential barrier (its height $\Delta_{m}$ depends on the top gate voltage $V_{t}$ and other voltages) for the electrons propagating between the contacts.

We shall assume that the GBL-FETs under the conditions when the electron systems in the source and drain sections are degenerate, i.e., $\varepsilon_{F} \gg k_{B} T$. This implies that the back gate voltage is sufficiently high to induce necessary electron density in the source and drain sections.
In the GBL-FET the energy gap is electrically induced by the back gate voltage [32 34] (see also [18]). Thus in GBL-FETs, the back gate plays the dual role: it provides the formation of the electron channel and the energy gap. Since the electric field component directed perpendicular to the GBL plane in the channel section below the top gate (gated section) is determined by both $V_{b}$ and $V_{t}$, the energy gap can be different in different sections of the GBL channel: $E_{g, s}$ (source section), $E_{g}$ (gated section), and $E_{g, d}$ (drain section) [17, 18]. At sufficiently strong top-gate voltage $\left(V_{t}<V_{t h}<0\right.$, where $V_{t h}$ is the threshold voltage), the gated section becomes depleted. Since the energy gaps in GBLs are in reality not particularly wide, at further moderate increase in $\left|V_{t}\right|$, the gated section of the channel becomes filled with holes (inversion of the charge in the gated section) if $V_{t}<V_{i n}<V_{t h}$, where $V_{i n}$ is the inversion voltage. As a result, the GBLFETs with moderate energy gap are characterized by the threshold and inversion voltages: $V_{t h}<0$ and $V_{i n}<0$. The explicit formulas for $V_{t h}$ and $V_{i n}$ shall be given in the following. The cases $V_{t}=V_{t h}$ and $V_{t}=V_{i n}$ correspond to the alignment of the Fermi level in the source section of the channel with the conduction band bottom and the valence band top, respectively, in the gated section.

\section{MAIN EQUATIONS OF THE MODEL}

Due to relatively high energy of optical phonons in graphene, the electron scattering in the GBL-FET channel is primarily due to disorder and acoustic phonons. Considering such quasielastic scattering, the quasiclassical Boltzmann kinetic equation governing the steady state electron distribution function $f_{\mathbf{p}}=f_{\mathbf{p}}(x)$ in the gated section of the channel can be presented as

$v_{x} \frac{\partial f_{\mathbf{p}}}{\partial x}+e \frac{\partial \varphi}{\partial x} \frac{\partial f_{\mathbf{p}}}{\partial p_{x}}=\int d^{2} \mathbf{q} w(q)\left(f_{\mathbf{p}+\mathbf{q}}-f_{\mathbf{p}}\right) \delta\left(\varepsilon_{\mathbf{p}+\mathbf{q}}-\varepsilon_{\mathbf{p}}\right)$.

Here $e=|e|$ is the electron charge, $\varepsilon_{\mathbf{p}}=p^{2} / 2 m, m \sim$ $0.04 m_{0}$ is the electron effective mass in GBL $\left(m_{0}\right.$ is the bare electron mass), $\mathbf{p}=\left(p_{x}, p_{y}\right)$ is the electron momentum in the GBL plane $(z=0), w(q)$ is the probability of the electron scattering on disorder and acoustic phonons with the variation of the electron momentum by quantity $\mathbf{q}=\left(q_{x}, q_{y}\right), v_{x}=p_{x}$, and axis $x$ is directed in this plane (from the source contact to the drain contact, i.e., in the direction of the current). For simplicity, we disregard the effect of "Mexican hat" (see, for instance, Ref. [34]) and a deviation of the real energy spectrum in the GBL from the parabolic one (the latter can be marked in the source and drain sections with relatively high Fermi energies). One of the potential advantages of GBL-FETs is the possibility of ballistic transport even if the top-gate length $L_{t}$ is not small. In such GBL-FETs, one can neglect the right-hand side term in Eq. (1).

As in Ref. [10, 15, 34, 36], we use the following equation for the electric potential $\varphi=\varphi(x)=\left.\psi(x, z)\right|_{z=0}$ in 
the GBL plane:

$$
\frac{\left(W_{b}+W_{t}\right)}{3} \frac{\partial^{2} \varphi}{\partial x^{2}}-\frac{\varphi-V_{b}}{W_{b}}-\frac{\varphi-V_{t}}{W_{t}}=\frac{4 \pi e}{k}\left(\Sigma_{-}-\Sigma_{+}\right) .
$$

Here, $\Sigma_{-}$and $\Sigma_{+}$are the electron and hole sheet densities in the channel, respectively, $k$ is the dielectric constant of the layers between the GBL and the gates, and $W_{b}$ and $W_{t}$ are the thicknesses of this layers. In the following, we put $W_{b}=W_{t}=W$ (except Sec. V). Equation (2) is a consequence of the two-dimensional Poisson equation for the electric potential $\psi(x, z)$ in the GBL-FET gated section $\left(-L_{t} / 2 \leq x \leq L_{t} / 2\right.$ and $-W_{b} \leq z \leq W_{t}$, where $L_{t}$ is the length of the top gate) in the weak nonlocality approximation [35]. This equation provides the potential distributions, which can be obtained from the two-dimensional Poisson equation by expansion in powers of the parameter $\delta=\left(W_{b}^{3}+W_{t}^{3}\right) / 15\left(W_{b}+W_{t}\right) / \mathcal{L}^{2}=W^{2} / 15 \mathcal{L}^{2}$, where $\mathcal{L}$ is the characteristic scale of the lateral inhomogeneities (in the $x$-direction) assuming that $\delta \ll 1$, i.e., $\mathcal{L}$ is not too small. The lowest approximation in such an expansion leads to the Shockley's gradual channel approximation, in which the first term in the left side of Eq. (2) is neglected [37, 38]. The factor $1 / 3$ appeared due to features of the Green function of the Laplace operator in the case of the geometry under consideration.

The boundary conditions for Eqs. (1) and (2) are presented as

$$
\begin{gathered}
\left.f_{\mathbf{p}}\right|_{p_{x} \geq 0, x=-L_{t} / 2}=f_{s, \mathbf{p}},\left.\quad f_{\mathbf{p}}\right|_{p_{x} \leq 0, x=L_{t} / 2}=f_{d, \mathbf{p}}, \quad(3) \\
\left.\varphi\right|_{x=-L_{t} / 2}=0,\left.\quad \varphi\right|_{x=L_{t} / 2}=V_{d}+\left(\varepsilon_{F, d}-\varepsilon_{F, s}\right) / e=V_{d}^{*},
\end{gathered}
$$

where $f_{s, \mathbf{p}}$ and $f_{d, \mathbf{p}}$ are the electron distribution functions in the source and drain sections of the channel. The functions $f_{s, \mathbf{p}}$ and $f_{d, \mathbf{p}}$ are the Fermi distribution functions with the Fermi energies $\varepsilon_{F, s}$ and $\varepsilon_{F, d}$, which are determined by the back gate and drain voltages, $V_{b}$ and $V_{d}$ [17, 18] (see also the Appendix):

$$
\varepsilon_{F, s} \simeq e V_{b} \frac{b}{(1+b)}, \quad \varepsilon_{F, d} \simeq e\left(V_{b}-V_{d}\right) \frac{b}{(1+b)},
$$

where $b=a_{B} / 8 W, a_{B}=k \hbar^{2} / m e^{2}$ is the Bohr radius, and $\hbar$ is the reduced Planck constant. In the following, we shall assume that $b \ll 1$, so that $\varepsilon_{F, s} \simeq b e V_{b}$ and $\varepsilon_{F, d} \simeq b e\left(V_{b}-V_{d}\right)$. In particular, if $a_{B}=4 \mathrm{~nm}$ (GBL on $\mathrm{SiO}_{2}$ ) and $W=10 \mathrm{~nm}$, one obtains $b \simeq 0.05$. Due to a smallness of $b$, we shall disregard a distinction between $V_{d}^{*}$ and $V_{d}$ because $V_{d}-V_{d}^{*} \simeq b V_{d} \ll V_{d}$ (as shown in the Appendix). Restricting ourselves by the consideration of GBL-FETs operation at not too high drain voltages, we also neglect the difference in the Fermi energies in the source and drain sections, i.e., put $\varepsilon_{F, d} \simeq \varepsilon_{F, s}=\varepsilon_{F}$.

The source-drain dc current density (current per unit length in the direction perpendicular its flow) can be calculated using the following formulae:

$$
\begin{gathered}
J=\frac{4 e}{(2 \pi \hbar)^{2}} \int d^{2} \mathbf{p} v_{x} f_{\mathbf{p}} \\
=\frac{e}{\pi^{2} \hbar^{2}} \int_{-\infty}^{\infty} d p_{y} \int_{0}^{\infty} d p_{x} v_{x}\left(f_{\mathbf{p}}-f_{-\mathbf{p}}\right) .
\end{gathered}
$$

In this case, Eq. (1) with boundary conditions (3) yield

$$
f_{\mathbf{p}}-f_{-\mathbf{p}} \simeq \frac{\Theta\left(p_{x}^{2} / 2 m+e \varphi\right)-\Theta\left(p_{x}^{2} / 2 m+e \varphi-e V_{d}\right)}{1+\exp \left[\left(p^{2} / 2 m+e \varphi-\varepsilon_{F}\right) / k_{B} T\right]}
$$

where $T$ is the temperature, $k_{B}$ is the Boltzmann constant, and $\Theta(\varepsilon)$ is the unity step function. Using Eqs. (6) and (7), we obtain

$$
\begin{aligned}
& J=\frac{e}{\pi^{2} \hbar^{2}} \int_{-\infty}^{\infty} d p_{y} \int_{\Delta_{m}}^{\infty} d \xi\left\{\frac{1}{1+\exp \left[\left(p_{y}^{2} / 2 m+\xi-\varepsilon_{F}\right) / k_{B} T\right]}-\frac{1}{1+\exp \left[\left(p_{y}^{2} / 2 m+\xi-\varepsilon_{F}+e V_{d}\right) / k_{B} T\right]}\right\} \\
& -=\frac{e k_{B} T}{\pi^{2} \hbar^{2}} \int_{-\infty}^{\infty} d p_{y}\left\{\ln \left[\exp \left(\frac{\varepsilon_{F}-p_{y}^{2} / 2 m-\Delta_{m}}{k_{B} T}\right)+1\right]-\ln \left[\exp \left(\frac{\varepsilon_{F}-p_{y}^{2} / 2 m-\Delta_{m}-e V_{d}}{k_{B} T}\right)+1\right]\right\} .
\end{aligned}
$$

Equation (8) can be presented in the following form:

$$
\begin{aligned}
J= & J_{0} \int_{0}^{\infty} d z\left\{\ln \left[\exp \left(\delta_{m}-z^{2}\right)+1\right]\right. \\
& \left.-\ln \left[\exp \left(\delta_{m}-U_{d}-z^{2}\right)+1\right]\right\} .
\end{aligned}
$$

Here (see, for instance, Ref. [23])

$$
J_{0}=\frac{2 \sqrt{2 m} e\left(k_{B} T\right)^{3 / 2}}{\pi^{2} \hbar^{2}}
$$

is the characteristic current density, and $\delta_{m}=\left(\varepsilon_{F}-\right.$ $\left.\Delta_{m}\right) / k_{B} T$, and $U_{d}=e V_{d} / k_{B} T$ are the normalized volt- 


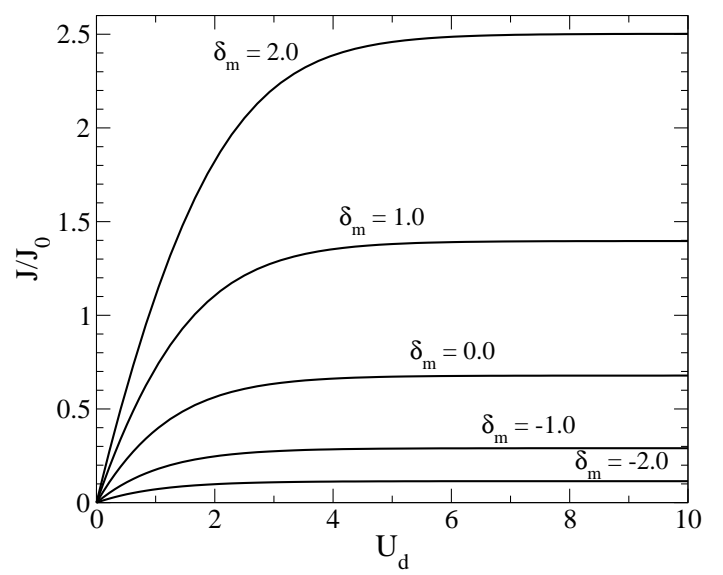

FIG. 2: Normalized source-drain current $J / J_{0}$ versus normalized drain voltage $U_{d}$ at different values of normalized gatevoltage swing $\delta_{m}$.

age swing and drain voltage, respectively. At $m=$ $4 \times 10^{-29} \mathrm{~g}$ and $T=300 \mathrm{~K}, J_{0} \simeq 2.443 \mathrm{~A} / \mathrm{cm}$.

Figure 2 shows the dependences of the source-drain current $J$ normalized by the value $J_{0}$ as a function of the $U_{d}$ calculated using Eq. (9) for different values of $\delta_{m}$.

The GBL-FET transconductance $g$ is defined as

$$
g=\frac{\partial J}{\partial V_{t}}
$$

Equations (9) and (11) yield

$$
\begin{gathered}
g=J_{0} \int_{0}^{\infty} d z\left\{\left[\exp \left(z^{2}-\delta_{m}\right)+1\right]^{-1}\right. \\
\left.-\left[\exp \left(z^{2}-\delta_{m}+U_{d}\right)+1\right]^{-1}\right\}\left(-\frac{\partial \delta_{m}}{\partial V_{t}}\right) .
\end{gathered}
$$

The obtained formulas for the source-drain current and transconductance can be simplified in the following limiting cases:

A. High top-gate voltages. At high top-gate voltages, which correspond to the sub-threshold voltage range, the barrier height exceeds the Fermi energy $\left(\Delta_{m} \gg \varepsilon_{F}\right)$, so that $\delta_{m} \gg 1$. In this case (the electron system in the gated section is nondegenerate), using Eqs. (9) and (12), we obtain

$$
J=\frac{\sqrt{\pi}}{2} J_{0} \exp \left(\frac{\varepsilon_{F}-\Delta_{m}}{k_{B} T}\right)\left[1-\exp \left(-\frac{e V_{d}}{k_{B} T}\right)\right]
$$

$g=\frac{\sqrt{\pi}}{2} \frac{J_{0}}{k_{B} T} \exp \left(\frac{\varepsilon_{F}-\Delta_{m}}{k_{B} T}\right)\left[1-\exp \left(-\frac{e V_{d}}{k_{B} T}\right)\right]\left(-\frac{\partial \Delta_{m}}{\partial V_{t}}\right)$.

B. Near threshold top-gate voltages. In this case, $\Delta_{m} \gtrsim$ $\varepsilon_{F}$, i.e., $\left|\delta_{m}\right| \lesssim 1$, Eqs. (9) and (12) yield

$$
\begin{gathered}
J \simeq J_{0} \frac{e V_{d}}{k_{B} T}\left[\zeta_{1}+\zeta_{2}\left(\frac{\varepsilon_{F}-\Delta_{m}}{k_{B} T}\right)\right]\left[1-\exp \left(-\frac{e V_{d}}{k_{B} T}\right)\right], \\
g \simeq \frac{J_{0} e V_{d}}{\left(k_{B} T\right)^{2}} \zeta_{2}\left(-\frac{\partial \Delta_{m}}{\partial V_{t}}\right)
\end{gathered}
$$

at low drain voltages $e V_{d} \lesssim k_{B} T\left(U_{d} \lesssim 1\right)$, and

$$
\begin{gathered}
J \simeq J_{0}\left[\zeta_{0}+\zeta_{1}\left(\frac{\varepsilon_{F}-\Delta_{m}}{k_{B} T}\right)\right], \\
g \simeq \frac{J_{0}}{k_{B} T} \zeta_{1}\left(-\frac{\partial \Delta_{m}}{\partial V_{t}}\right)
\end{gathered}
$$

at high drain voltages $e V_{d} \gg k_{B} T\left(U_{d} \gg 1\right)$. Here, $\zeta_{0}=$ $\int_{0}^{\infty} d \xi \ln \left[\exp \left(-\xi^{2}\right)+1\right] \simeq 0.678, \zeta_{1}=\int_{0}^{\infty} d \xi /\left[\exp \left(\xi^{2}\right)+\right.$ $1] \simeq 0.536$, and $\zeta_{2}=\int_{0}^{\infty} d \xi \exp \left(\xi^{2}\right) /\left[\exp \left(\xi^{2}\right)+1\right]^{2}$. In the limit $\varepsilon_{F}=\Delta_{m}$, Eqs. (13) and (14) provide the values $J$ and $g$ close to those obtained from Eqs. (17) and (18), which are rigorous in such a limit.

C. Low top-gate voltages. At low top-gate voltages, $\Delta_{m}<\varepsilon_{F}$, from Eqs. (9)

$$
J \simeq \frac{2}{3} I_{0}\left[\left(\varepsilon_{F}-\Delta_{m}\right)^{3 / 2} \Theta\left(\varepsilon_{F}-\Delta_{m}\right)\right.
$$

$$
\begin{gathered}
\left.-\left(\varepsilon_{F}-\Delta_{m}-e V_{d}\right)^{3 / 2} \Theta\left(\varepsilon_{F}-\Delta_{m}-e V_{d}\right)\right] . \\
g \simeq I_{0}\left[\left(\varepsilon_{F}-\Delta_{m}\right)^{1 / 2} \Theta\left(\varepsilon_{F}-\Delta_{m}\right)\right. \\
\left.-\left(\varepsilon_{F}-\Delta_{m}-e V_{d}\right)^{1 / 2} \Theta\left(\varepsilon_{F}-\Delta_{m}-e V_{d}\right)\right]\left(-\frac{\partial \Delta_{m}}{\partial V_{t}}\right) .
\end{gathered}
$$

Here,

$$
I_{0}=\frac{2 \sqrt{2 m} e}{\pi^{2} \hbar^{2}},
$$

with $J_{0}=I_{0}\left(k_{B} T\right)^{3 / 2}$.

Using Eqs. (19) and (20), one obtains

$$
J \simeq I_{0} e V_{d} \sqrt{\left(\varepsilon_{F}-\Delta_{m}\right)} \simeq I_{0} e V_{d} \sqrt{\varepsilon_{F}}\left(1-\frac{\Delta_{m}}{2 \varepsilon_{F}}\right),
$$

$$
g \simeq \frac{1}{2} I_{0} \frac{e V_{d}}{\sqrt{\varepsilon_{F}}}\left(-\frac{\partial \Delta_{m}}{\partial V_{t}}\right)
$$

at $e V_{d} \ll \varepsilon_{F}-\Delta_{m}$, and

$$
J=\frac{2}{3} I_{0}\left(\varepsilon_{F}-\Delta_{m}\right)^{3 / 2} \simeq \frac{2}{3} I_{0} \varepsilon_{F}^{3 / 2}\left(1-\frac{3 \Delta_{m}}{2 \varepsilon_{F}}\right),
$$




$$
g \simeq I_{0} \sqrt{\left(\varepsilon_{F}-\Delta_{m}\right)}\left(-\frac{\partial \Delta_{m}}{\partial V_{t}}\right)
$$

at $e V_{d} \gg \varepsilon_{F}-\Delta_{m}$.

The dependences shown in Fig. 2 describe implicitly the dependences of $J$ calculated using the universal Eq. (9) on the back-gate, top-gate, and drain voltages as well as on the geometrical parameters. Equations (13) (25) provide these dependences in most interesting limits. However, to obtain the explicit formulas for $J$ as well as for $g$, one needs to determine the dependences of the barrier height $\Delta_{m}$ on all voltages and geometrical parameters. Since the electron densities in the gated section in the limiting cases under consideration are different, the screening abilities of the electron system in this section and the potential distributions are also different. The latter leads to different $\Delta_{m}$ vs $V_{t}$ relations.

\section{POTENTIAL DISTRIBUTIONS, SOURCE-DRAIN CURRENT, AND TRANSCONDUCTANCE}

To obtain the explicit dependences of the source-drain current and the transconductance on the gate voltages $V_{b}$ and $V_{t}$ as well as on the drain voltage $V_{d}$, one needs to find the relationship between the barrier height $\Delta_{m}$ and these voltages. This necessitates the calculations of the potential distribution in the channel. The latter can be found from Eq. (2) in an analytical form in the following limiting cases.

\section{A. High top-gate voltages - sub-threshold voltage range $\left(\Delta_{m} \gg \varepsilon_{F}\right)$.}

When the barrier height $\Delta_{m}$ exceeds the Fermi energy $\varepsilon_{F}$, the electron density is low in the gated section and, hence, one can disregard the contribution of the electron charge in this section. In such a limit, we arrive at the following equation for the potential:

$$
\frac{d^{2} \varphi}{d x^{2}}-\frac{\varphi}{\Lambda_{0}^{2}}=\frac{F_{0}}{\Lambda_{0}^{2}},
$$

where $\Lambda_{0}=\sqrt{2 / 3} W$ and $F_{0}=-\left(V_{b}+V_{t}\right) / 2$. Solving Eq. (26) considering boundary conditions (4), for the case of high top-gate voltages we obtain

$$
\varphi=F_{0}\left[\frac{\cosh \left(x / \Lambda_{0}\right)}{\cosh \left(L_{t} / 2 \Lambda_{0}\right)}-1\right]+V_{d} \frac{\sinh \left[\left(2 x+L_{t}\right) / 2 \Lambda_{0}\right]}{\sinh \left(L_{t} / \Lambda_{0}\right)} .
$$

Limiting our consideration by the GBL-FETs with not too short top gate $\left(L_{t} \gg W\right)$, Eq. (27) can be presented as

$\varphi \simeq-F_{0}\left[1-2 \exp \left(-\frac{L_{t}}{2 \Lambda_{0}}\right) \cosh \left(\frac{x}{\Lambda}\right)\right]+V_{d} \exp \left(-\frac{L_{t}}{2 \Lambda_{0}}\right) \exp$
Equation (28) yields

$\Delta_{m} \simeq e F_{0}\left(1-\frac{1}{\eta_{0}}\right)-\frac{e V_{d}}{2 \eta_{0}}=-\frac{e\left(V_{b}+V_{t}\right)}{2}\left(1-\frac{1}{\eta_{0}}\right)-\frac{e V_{d}}{2 \eta_{0}}$,

where $\eta_{0}=\exp \left(L_{t} / 2 \Lambda_{0}\right) / 2$. Simultaneously for the position of the barrier top one obtains

$$
x_{m}=-\frac{\Lambda_{0}}{2} \ln \left(1+\frac{V_{d}}{F_{0}}\right)=-\frac{\Lambda_{0}}{2} \ln \left(1-\frac{2 V_{d}}{V_{b}+V_{t}}\right) .
$$

The terms in the right-hand side of Eq. (29) containing parameter $\eta_{0}$ reflect the effect of the top-gate geometry (finiteness of its length). This effect is weakened with increasing top barrier length $L_{t}$. The effect of draininduced barrier lowering in the case under consideration is described by the last term in the right-hand side of Eq. (29).

Equation $(29)$ yields $\left(\partial \Delta_{m} / \partial V_{t}\right)=-(e / 2)\left(1-\eta_{0}^{-1}\right)$. Invoking Eqs. (13) and (14), we obtain

$$
\begin{gathered}
J=\frac{\sqrt{\pi}}{2} J_{0} \exp \left[\frac{e\left(V_{t}-V_{t h}\right)}{2 k_{B} T}\left(1-\frac{1}{\eta_{0}}\right)\right] \\
\times\left[1-\exp \left(-\frac{e V_{d}}{k_{B} T}\right)\right] \exp \left(\frac{e V_{d}}{2 \eta_{0} k_{B} T}\right), \\
g \simeq \frac{\sqrt{\pi}}{4} \frac{e J_{0}}{k_{B} T} \exp \left[\frac{e\left(V_{t}-V_{t h}\right)}{2 k_{B} T}\left(1-\frac{1}{\eta_{0}}\right)\right] \\
\quad \times\left[1-\exp \left(-\frac{e V_{d}}{k_{B} T}\right)\right] \exp \left(\frac{e V_{d}}{2 \eta_{0} k_{B} T}\right) .
\end{gathered}
$$

Here, $V_{t h}=-\left[1+2 b /\left(1-\eta_{0}^{-1}\right)\right] V_{b} \simeq-(1+2 b) V_{b}$. The rightmost factors in the right-hand sides of Eqs. (31) and (32), associated with the effect of drain-induced barrier lowering, lead to an increase in $g$ with increasing $V_{d}$ not only at $e V_{d} \sim k_{B} T$ but at $e V_{d} \gg k_{B} T$ : $g \propto \exp \left(e V_{d} / 2 \eta_{0} k_{B} T\right)$. One can see that in the range of the top-gate voltages under consideration, the GBLFET transconductance exponentially decreases with increasing $\left|V_{t}+V_{b}\right|$ and

$$
g \simeq \frac{J e}{2 k_{B} T} \ll g_{0}=\frac{\sqrt{\pi}}{4} \frac{e J_{0}}{k_{B} T},
$$

where at $T=300 \mathrm{~K}$ the characteristic value of the transconductance $g_{0} \simeq 4330 \mathrm{mS} / \mathrm{mm}$.

B. Near threshold top-gate voltages $\left(\Delta_{m} \gtrsim \varepsilon_{F}\right)$. $\left(\overline{A_{d} t}\right) \dot{e} V_{d} \lesssim k_{B} T \ll \varepsilon_{F}$, taking into account that the electron distribution is characterized by the equilibrium 
Fermi distribution function, the electron density in the gated section can be presented in the following form:

$$
\Sigma \simeq \frac{2 m}{\pi \hbar^{2}}\left(\varepsilon_{F}+e \varphi\right) .
$$

Considering this, we reduce Eq. (2) to

$$
\frac{d^{2} \varphi}{d x^{2}}-\frac{\varphi}{\Lambda^{2}}=\frac{F}{\Lambda^{2}} .
$$

Here,

$$
\begin{gathered}
\Lambda=\sqrt{\frac{a_{B} W}{12(1+2 b)}} \simeq \sqrt{\frac{a_{B} W}{12}}=W \sqrt{\frac{2}{3} b,} \\
F=\frac{\left[\varepsilon_{F} / e-b\left(V_{b}+V_{t}\right)\right]}{(1+2 b)} \simeq-b\left(b V_{b}+V_{t}\right) \simeq-b V_{t},
\end{gathered}
$$

so that $\Lambda / \Lambda_{0} \simeq \sqrt{b}<1$. The solution of Eq. (35) with boundary condition (4) is given by

$$
\begin{gathered}
\varphi=F\left[\frac{\cosh (x / \Lambda)}{\cosh \left(L_{t} / 2 \Lambda\right)}-1\right]+V_{d} \frac{\sinh \left[\left(2 x+L_{t}\right) / 2 \Lambda\right]}{\sinh \left(L_{t} / \Lambda\right)} \\
\simeq-F\left[1-2 \exp \left(-\frac{L_{t}}{2 \Lambda}\right) \cosh \left(\frac{x}{\Lambda}\right)\right] \\
+V_{d} \exp \left(-\frac{L_{t}}{2 \Lambda}\right) \exp \left(\frac{x}{\Lambda}\right)
\end{gathered}
$$

From Eq. (36) we obtain

$$
\begin{gathered}
\Delta_{m} \simeq e F\left(1-\frac{1}{\eta}\right)-\frac{e V_{d}}{2 \eta} \\
\simeq\left[\varepsilon_{F}-e b\left(V_{b}+V_{t}\right)\right]\left(1-\frac{1}{\eta}\right)-\frac{e V_{d}}{2 \eta} \simeq-e b V_{t}\left(1-\frac{1}{\eta}\right)-\frac{e V_{d}}{2 \eta},
\end{gathered}
$$

where $\eta=\exp \left(L_{t} / 2 \Lambda\right) / 2$, and the position of the barrier top is

$$
x_{m} \simeq-\frac{\Lambda}{2} \frac{V_{d}}{F} \simeq-\frac{\Lambda}{2 b} \frac{V_{d}}{V_{b}} .
$$

Since $\Lambda<\Lambda_{0}$, one obtains $\eta \gg \eta_{0}$, and the terms in Eq. (37) containing parameter $\eta$ can be disregarded. This implies that the effects of top-gate geometry and draininduced barrier lowering are much weaker (negligible) in the case of the top-gate voltages in question in comparison with the case of high top-gate voltages.

Substituting $\Delta_{m}$ from Eq. (37) into Eq. (16), for low drain voltages we arrive at

$$
g \simeq \frac{J_{0} e^{2} V_{d} \zeta_{2}}{\left(k_{B} T\right)^{2}}\left(1-\frac{1}{\eta}\right) b .
$$

At relatively high drain voltages $\left(e V_{d}>\varepsilon_{F} \gg k_{B} T\right)$, the electron charge in the source portion of the gated section $\left(x \leq x_{m}\right.$, where $x_{m}$ is the coordinate of the barrier top) is primarily determined by the electrons injected from the source. The electron injection from the drain at high drain voltages is insignificant. Hence, the electron charge in the drain portion of the gated section can be disregarded. In this case, Eq. (2) can be presented as

$$
\frac{d^{2} \varphi}{d x^{2}}-\frac{\varphi}{\Lambda^{2}}=\frac{F}{\Lambda^{2}}
$$

at $-L_{t} / 2 \leq x \leq x_{0}$, and

$$
\frac{d^{2} \varphi}{d x^{2}}-\frac{\varphi}{\Lambda_{0}^{2}}=\frac{F_{0}}{\Lambda_{0}^{2}}
$$

at $x_{0} \leq x \leq L_{t} / 2$.

At the point $x=x_{0}$ corresponding to the condition $\left.e \varphi\right|_{x=x_{0}}+\varepsilon_{F}=0$, the solutions of Eqs. (40) and (41) should be matched:

$\left.\varphi\right|_{x=x_{0}-0}=\left.\varphi\right|_{x=x_{0}+0}=-\frac{\varepsilon_{F}}{e},\left.\quad \frac{d \varphi}{d x}\right|_{x=x_{0}-0}=\left.\frac{d \varphi}{d x}\right|_{x=x_{0}+0}$.

Solving Eqs. (40) and (41) with conditions (4) and (42), we obtain the following formulas for the potential $\varphi$ at $-L_{t} / 2 \leq x \leq x_{0}$ and $x_{0} \leq x \leq L_{t} / 2$ as well as an equation for $x_{0}$ :

$$
\begin{gathered}
\varphi=F\left[\frac{\cosh (x / \Lambda)}{\cosh \left(L_{t} / 2 \Lambda\right)}-1\right]-\left[\frac{\varepsilon_{F}}{e}+F \frac{\cosh \left(x_{0} / \Lambda\right)}{\cosh \left(L_{t} / 2 \Lambda\right)}-F\right] \frac{\left.\sinh \left[\left(x+L_{t} / 2\right) / \Lambda\right)\right]}{\left.\sinh \left[\left(x_{0}+L_{t} / 2\right) / \Lambda\right)\right]} \\
\varphi=F_{0}\left[\frac{\cosh \left(x / \Lambda_{0}\right)}{\cosh \left(L_{t} / 2 \Lambda_{0}\right)}-1\right]+V_{d} \frac{\sinh \left[\left(x+L_{t} / 2\right) / \Lambda_{0}\right]}{\sinh \left(L_{t} / \Lambda_{0}\right)} \\
-\left[\frac{\varepsilon_{F}}{e}+F_{0} \frac{\cosh \left(x_{0} / \Lambda_{0}\right)}{\cosh \left(L_{t} / 2 \Lambda_{0}\right)}-F_{0}+V_{d} \frac{\sinh \left[\left(x_{0}+L_{t} / 2\right) / \Lambda_{0}\right]}{\sinh \left(L_{t} / \Lambda_{0}\right)}\right] \frac{\sinh \left[\left(x-L_{t} / 2\right) / \Lambda_{0}\right]}{\sinh \left[\left(x_{0}-L_{t} / 2\right) / \Lambda_{0}\right]}
\end{gathered}
$$




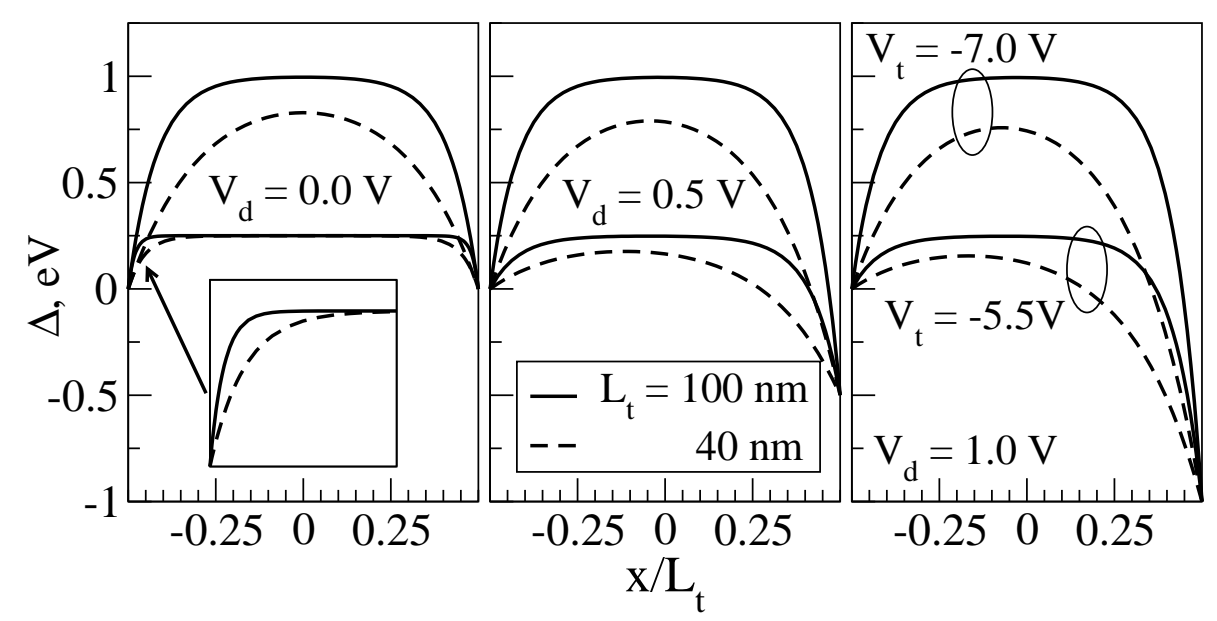

FIG. 3: Barrier profile $\Delta=-e \varphi$ at different top-gate voltage $V_{t}$ and drain voltage $V_{d}$ for GBL-FETs with different top-gate length $L_{t}$. Upper and lower pairs of curves correspond to $V_{t}-V_{t h}=-1.5 \mathrm{~V}$ and $V_{t}-V_{t h} \simeq 0$, respectively; $W=10 \mathrm{~nm}, b=0.05$, and $V_{b}=5.0 \mathrm{~V}$.

$$
\begin{gathered}
\frac{1}{\Lambda}\left\{F \frac{\sinh \left(x_{0} / \Lambda\right)}{\cosh \left(L_{t} / 2 \Lambda\right)}-\left[\frac{\varepsilon_{F}}{e}+F \frac{\cosh \left(x_{0} / \Lambda\right)}{\cosh \left(L_{t} / 2 \Lambda\right)}-F\right] \frac{\left.\cosh \left[\left(x_{0}+L_{t} / 2\right) / \Lambda\right)\right]}{\left.\sinh \left[\left(x_{0}+L_{t} / 2\right) / \Lambda\right)\right]}\right\} \\
=\frac{1}{\Lambda_{0}}\left\{F_{0} \frac{\sinh \left(x_{0} / \Lambda_{0}\right)}{\cosh \left(L_{t} / 2 \Lambda_{0}\right)}+V_{d} \frac{\cosh \left[\left(x_{0}+L_{t} / 2\right) / \Lambda_{0}\right]}{\sinh \left(L_{t} / \Lambda_{0}\right)}\right. \\
\left.-\left[\frac{\varepsilon_{F}}{e}+F_{0} \frac{\cosh \left(x_{0} / \Lambda_{0}\right)}{\cosh \left(L_{t} / 2 \Lambda_{0}\right)}-F_{0}+V_{d} \frac{\sinh \left[\left(x_{0}+L_{t} / 2\right) / \Lambda_{0}\right]}{\sinh \left(L_{t} / \Lambda_{0}\right)}\right] \frac{\cosh \left[\left(x_{0}-L_{t} / 2\right) / \Lambda_{0}\right]}{\sinh \left[\left(x_{0}-L_{t} / 2\right) / \Lambda_{0}\right]}\right\} .
\end{gathered}
$$

In the cases $V_{b}+V_{t} \simeq 0$ and $-\left(V_{b}+V_{t}\right) \gtrsim V_{b} \gg \varepsilon_{F} / e$, Eq. (45) yields $x_{0}=-L_{t} / 2+\Lambda \ln \left[4 b V_{t} /(\sqrt{b}+2 b)\left(V_{b}+\right.\right.$ $\left.\left.V_{t}\right)\right]$ and $x_{0} \simeq-L_{t} / 2+2 \Lambda_{0} \varepsilon_{F} /\left[-e\left(V_{b}+V_{t}\right)\right] \simeq-L_{t} / 2+$ $2 b \Lambda_{0} V_{b} /\left[-\left(V_{b}+V_{t}\right)\right]$, respectively. When $-\left(V_{b}+V_{t}\right) \rightarrow$ +0 , the matching point shifts toward the channel center. If $-\left(V_{b}+V_{t}\right)$ increases, the matching point tends to the the source edge of the channel. In this case, the role of the electron charge in the vicinity of the source edge diminishes, and the potential distribution tends to that given by Eq. (27).

At the threshold, the matching point $x_{0}$ coincides with the position of the barrier maximum $x_{m}$. Considering that at $x_{0}=x_{m}$, both the left-hand and right-hand sides of Eq. (45) are equal to zero, for the barrier top height near the threshold at relatively high drain voltages we obtain the following:

$\Delta_{m} \simeq e F_{0}\left(1-\frac{1}{\eta_{0}}\right)-\frac{e V_{d}}{\eta_{0}}=-\frac{e\left(V_{b}+V_{t}\right)}{2}\left(1-\frac{1}{\eta_{0}}\right)-\frac{e V_{d}}{\eta_{0}}$

$$
x_{m} \simeq-\frac{L_{t}}{2}+\Lambda \ln \left(\frac{2 F}{F-F_{0}}\right) \simeq-\frac{L_{t}}{2}+\Lambda \ln \frac{1}{b} .
$$

Both Eqs. (29) and (46) correspond to the situations when the electron density in a significant portion of the channel is fairly low. However there is a distinction in the dependence of $\Delta_{m}$ on $V_{d}$ (the pertinent coefficients differ by factor of two). This is because in the first case the barrier top is located near the channel center, whereas in the second case it is shifted to the vicinity of the source edge [compare Eqs. (30) and (47)].

Using Eqs. (19) and (50), we obtain $\left(\partial \Delta_{m} / \partial V_{t}\right)=$ $-(e / 2)\left(1-\eta_{0}^{-1}\right)$ and arrive at the following formula for the transconductance near the threshold, i.e., when $V_{t} \simeq V_{t h}$

$$
J \simeq J_{0}\left[\zeta_{0}+\zeta_{1} \frac{e\left(V_{t}-V_{t h}\right)}{2 k_{B} T}\left(1-\frac{1}{\eta_{0}}\right)\right],
$$

$$
g \simeq \frac{J_{0} e}{k_{B} T} \frac{\zeta_{1}}{2}\left(1-\frac{1}{\eta_{0}}\right)=g_{t h} .
$$


Here, as above, $V_{t h} \simeq-(1+2 b) V_{b}$. In particular, Eqs. (48) and (49) at $V_{t}=V_{t h}$, yield $J_{t h} \simeq J_{0} \zeta_{0}$. For a GBL-FET with $L_{t}=40 \mathrm{~nm}, W=10 \mathrm{~nm}$, at $T=300 \mathrm{~K}$, one obtains $J_{t h} \simeq 1.656 \mathrm{~A} / \mathrm{cm}$ and $g_{t h} \simeq 2167 \mathrm{mS} / \mathrm{mm}$.

\section{Low top-gate voltages $\left(\Delta_{m}<\varepsilon_{F}\right)$.}

At relatively low top-gate voltages when $\Delta_{m}<\varepsilon_{F}$, the electron system is degenerate not only in the source and drain sections but in the gate section as well. In this top-gate voltage range, the spatial variation of the potential is characterized by $\Lambda \simeq W \sqrt{2 b / 3}$. As a result, for $\Delta_{m}$ one obtains an equation similar to Eq. (37). Since $\Lambda<\Lambda_{0} \ll L_{t}$, the parameter determining the effect of the top-gate geometry and the effect of drain-induced barrier lowering is $\eta=\exp \left(L_{t} / 2 \Lambda\right) / 2 \gg \eta_{0}$. As a consequence, one can neglect the effects in question in the top-gate voltage range under consideration. In this case, $\varepsilon_{F}-$ $\Delta_{m} \simeq b V_{t}$ is As a result, one can arrive at

$$
J \simeq \frac{2}{3} I_{0} e^{3 / 2}\left[b\left(V_{t}-V_{t h}\right)\right]^{3 / 2}-\left[b\left(V_{t}-V_{t h}-V_{d}\right]^{3 / 2}\right.
$$

when $V_{d} \leq b\left(V_{t}-V_{t h}\right)$,

$$
J \simeq \frac{2}{3} I_{0} e^{3 / 2}\left[b\left(V_{t}-V_{t h}\right)\right]^{3 / 2},
$$

when $V_{d}>b\left(V_{t}-V_{t h}\right)$,

$$
\frac{\partial \Delta_{m}}{\partial V_{t}}=-b e
$$

Considering Eq. (51), at low top-gate voltages we obtain

$$
g \simeq \frac{b e I_{0}}{2} \frac{e V_{d}}{\sqrt{\varepsilon_{F}}} \simeq \frac{\sqrt{b} e^{3 / 2} I_{0}}{2} \frac{V_{d}}{\sqrt{V_{b}}}
$$

when $e V_{d} \ll \varepsilon_{F}-\Delta_{m} \simeq b e\left(V_{b}+V_{t}\right)$, and

$$
g \lesssim b e I_{0} \sqrt{\varepsilon_{F}} \simeq b^{3 / 2} e^{3 / 2} I_{0} \sqrt{V_{b}}=g_{\text {on }}
$$

when $e V_{d} \gg \varepsilon_{F}-\Delta_{m} \simeq b e\left(V_{b}+V_{t}\right)$. As follows from Eqs. (33) and (34), the transconductance is proportional to a small parameter $b^{3 / 2}$. This is because the effect of the top-gate potential is weakened due to a strong screening by the degenerate electron system in the gated section. As a result, the transconductance at low top-gate voltages is smaller than that at the topgate voltage corresponding to the threshold. Assuming that $b=0.05$ and $V_{b}=5\left(\varepsilon_{F} \simeq 0.25 \mathrm{eV}\right)$, we obtain $g_{\text {on }} \simeq 1467 \mathrm{mS} / \mathrm{mm}$. Comparing Eqs. (49) and (54), we find $g_{\text {on }} / g_{t h} \propto b^{3 / 2} \sqrt{e V_{b} / k_{B} T} \simeq 0.158$ and $g / g_{t h} \lesssim g_{\text {on }} / g_{t h} \simeq 0.68$.

Since the source-drain current at high top-gate voltages decreases exponentially when $-V_{t}$ increases, the transconductance decreases as well.

Figure 3 shows the barrier (conduction band) profile $\Delta=-e \varphi$ in the GBL-FETs calculated using Eqs. (27),

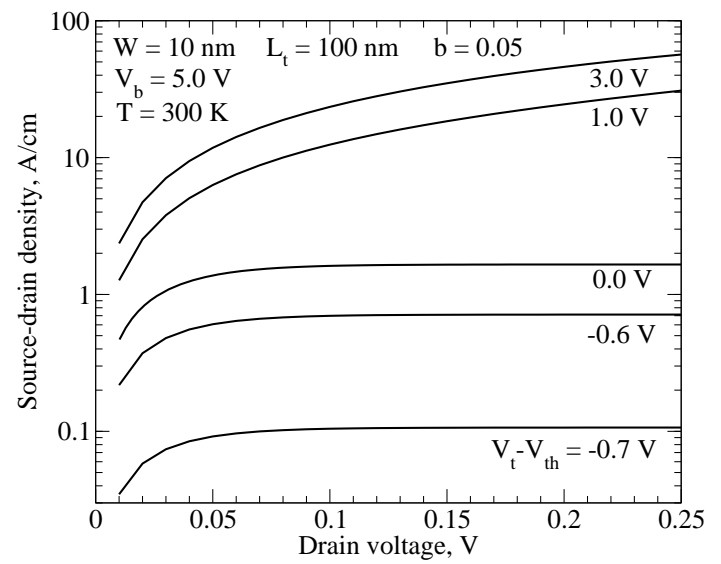

FIG. 4: Source-drain current $J$ versus drain voltage $V_{d}$ at different values of the top-gate voltage swing $V_{t}-V_{t h}$.

(36), and (44) for different applied voltages and top-gate lengths. As demonstrated, the barrier heigh naturally decreases with increasing $V_{t}-V_{t h}$. At the threshold $\left(V_{t}=V_{t h}\right)$, the barrier heigh is equal to the Fermi energy (at $b=0.05$ and $V_{b}=5 \mathrm{~V}, \varepsilon_{F} \simeq 0.25 \mathrm{eV}$ ). One can see that shortening of the top-gate leads to a marked decrease in the barrier height (the short-gate effect). The source-drain current as a function of the drain voltage for different top-gate voltage swings is demonstrated in Fig. 4. The dependences corresponding to $V_{t}-V_{t h}<0$, $V_{t}-V_{t h}=0$, and $V_{t}-V_{t h}>0$ were calculated using formulas from subsections A, B, and C, respectively. Figure 5 shows that the transconductance as a function of the top-gate voltage swing exhibits a pronounced maximum at $V_{t} \simeq V_{t h}$. This maximum is attributed to the following. At high top-gate voltages, the effect of screening is insignificant due to low electron density in the channel. As a result, the height of the barrier top is rather sensitive to the top-gate voltage variations. The sourcedrain current in this case is exponentially small, so that the transconductance is small. In contrast, at low topgate voltages, the screening by the electrons in the channel is effective, leading to a much weaker control of the barrier height by the top voltage [pay attention to parameter $b \ll 1$ in Eqs. (50) - (54)]. Despite, a strong source-drain current provides a moderate values of the transconductance. However, in the near threshold voltage range, both the sensitivity of the barrier height to the top-gate volatage and the source-drain current are fairly large.

\section{DISCUSSION}

\section{A. Role of geometrical parameters}

In the main part of the paper, we assumed that the thicknesses of the gate layers are equal to each other: 


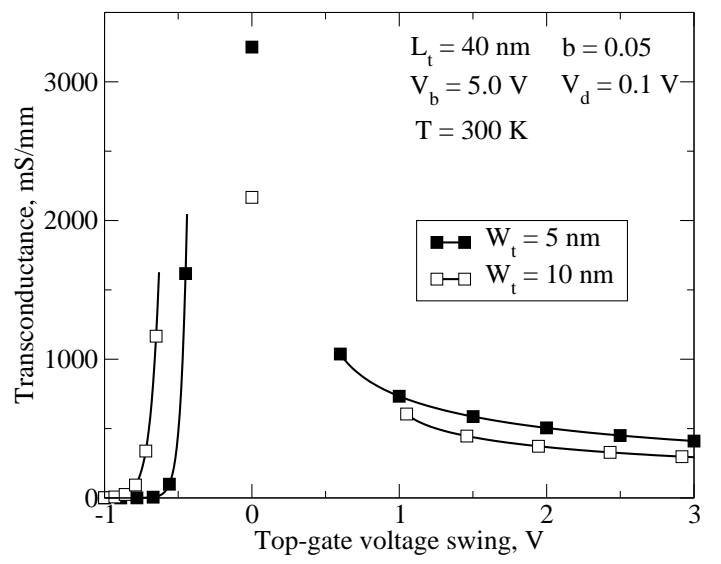

FIG. 5: Transconductance $g$ versus top-gate voltage swing $V_{t}-V_{t h}$

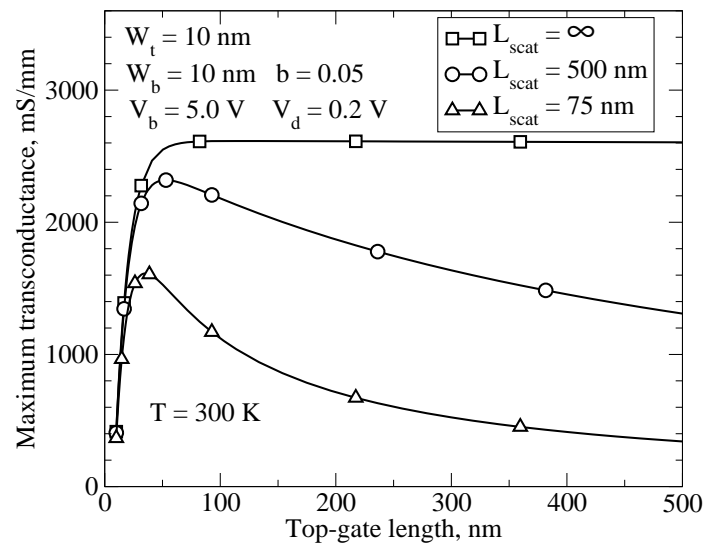

FIG. 6: Maximum transconductance $g$ versus top-gate length $L_{t}$ for $W_{t}=10 \mathrm{~nm}$.

$W_{b}=W_{t}=W$. If $W_{b} \neq W_{t}$, the formulas obtained above should be slightly modified. In particular, near the threshold $\left(\partial \Delta_{m} / \partial V_{t}\right)=-\left(e /\left(1+W_{t} / W_{b}\right)\right)\left(1-\eta_{0}^{-1}\right)$, so that the transconductance instead of Eq. (49) is given by

$$
\begin{gathered}
g \simeq \frac{J_{0} e}{k_{B} T} \frac{\zeta_{1}}{\left(1+W_{t} / W_{b}\right) \zeta_{0}}\left(1-\frac{1}{\eta_{0}}\right) \\
\simeq \frac{J_{0} e}{k_{B} T} \frac{\zeta_{1}}{\left(1+W_{t} / W_{b}\right) \zeta_{0}} .
\end{gathered}
$$

As follows from the comparison of Eqs. (49) and (55), changing $W_{t}$, in particular, from $W_{t}=W_{b}$ to $W_{t}=W_{b} / 2$, leads to an increase in the transconductance at the topgate voltages near the threshold of 50 percent (compare the $g$ versus $V_{t}-V_{t h}$ dependences in Fig. 5 for $W_{b}=$ $W_{t}=10 \mathrm{~nm}$ and $W_{b}=10 \mathrm{~nm}$ and $W_{t}=5 \mathrm{~nm}$.

As demonstrated above, shortening of the top gate can result in deterioration of the GBL-FET characteristics. This effect is characterized by parameters $\eta$ and

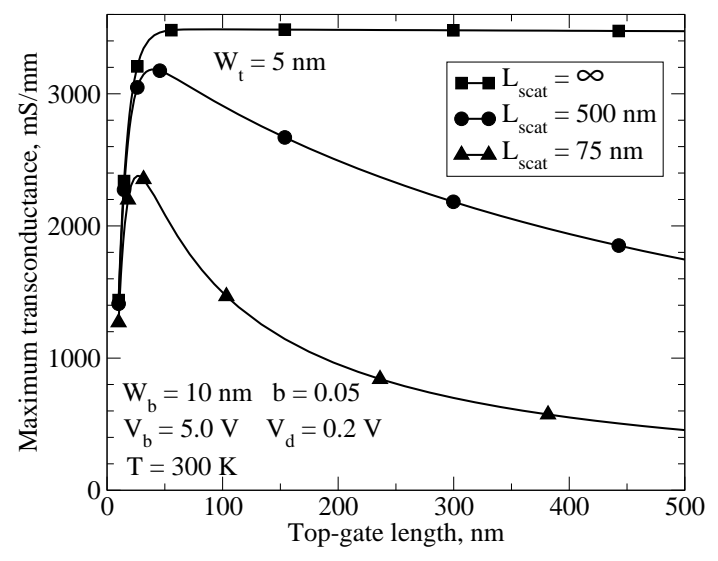

FIG. 7: The same as in Fig. 6 but for $W_{t}=5 \mathrm{~nm}$.

$\eta_{0}$, which strongly depends on the top-gate length $L_{t}$. This effect gives rise to a decrease in the transconductance if $L_{t}$ becomes smaller. However, in the range of large $L_{t}$ the electron collisions can play a substantial role. This leads to the transconductance roll-off with increasing $L_{t}$. The effect of drain-induced barrier lowering is also characterized by parameters $\eta$ and $\eta_{0}$. As follows from Sec. IV, at sub-threshold, the effect of draininduced barrier lowering results in the appearance of the factor $\exp \left(e V_{d} / 2 \eta_{0} k_{B} T\right)$ [see Eqs. (31) and (32)]. This factor can provide a marked increase in $J$ and $g$ with increasing drain voltage in GBL-FETs with relatively short top gates. For example, at $W=40 \mathrm{~nm}, L_{t}=40 \mathrm{~nm}$, $T=300 \mathrm{~K}$, and $V_{d}=0.25 \mathrm{~V}$, this factor is about 2.37.

\section{B. Effect of electron scattering}

As shown above, the potential distributions in the main part of the gated section are fairly flat. This implies that to determine the effect of electron scattering associated with disorder and acoustic phonons one can use Eq. (2) with the collisional term following the approach applied in Ref. [17]. Considering here the case when the elastic scattering mechanisms under consideration are strong, so that they lead to an effective isotropization of the electron distribution, one can find that the values of the source-drain current and the transconductance obtained in the previous section for the ballistic transport should be multiplied by a collision factor $C$. This factor is equals to $C_{\infty}=\sqrt{2 \pi k_{B} T / m} / L_{t} \nu$, where $\nu=m w / 2$ is the collision frequency (we put $w(q)=w=$ const. It characterizes the fraction of the electrons injected into the gated section and those reflected back due to the collisions. To obtain the GBL-FET characteristics with the top-gate lengths in a wide range (to follow the transition from the ballistic electron transport to the collisiondominated transport), we use for the collision factor the following interpolation formula: 


$$
C=\frac{1}{1+L_{t} / L_{\text {scat }}}
$$

where $L_{\text {scat }}=\sqrt{2 \pi k_{B} T / m} / \nu$ is the characteristic scattering length. Figures 6 and 7 show the dependences of the transconductance maximum (approximately at $\left.V_{t}=V_{t h}\right)$ on the top-gate length calculated for GBLFETs with different $W_{t}$. The scattering length is assumed to be $\infty$ (ballistic transport), $500 \mathrm{~nm}$, and $75 \mathrm{~nm}$. At $T=300 \mathrm{~K}$, this corresponds to the collision frequencies $\nu=0, \nu \simeq 1.14 \times 10^{12} \mathrm{~s}^{-1}$ (the electron mobilities $\left.\mu \simeq 1.75 \times 10^{5} \mathrm{~cm}^{2} / \mathrm{V} \mathrm{s}\right)$ and $\nu \simeq 7.6 \times 10^{12} \mathrm{~s}^{-1}$ $\left(\mu \simeq 2.63 \times 10^{4} \mathrm{~cm}^{2} / \mathrm{V} \mathrm{s}\right)$, respectively). One can see that in the case of essential electron collisions, $g$ versus $L_{t}$ dependences exhibit pronounced maxima. This is attributed to an interplay of two effects: the short-gate effect (weakening of the barrier controllability by the topgate voltage when $L_{t}$ decreases) and the effect of collisions, which reinforces when $L_{t}$ becomes larger. (which decreases the current). As follows from Figs. 6 and 7, the electron collisions can lead to a dramatic decrease in the transconductance.

\section{Charge inversion in the gated section.}

At sufficiently high top-gate voltages $V_{t}<V_{\text {in }}$ when $\Delta_{m}>\varepsilon_{F}+E_{g}$, the top of the valence band in the gated section of the channel can be markedly populated by holes (inversion of the gated section charge), so that the term in the right-hand side of Eq. (2) becomes negative. The latter inequality corresponds to the following value of the inversion voltage [see Eqs.(A4) and (A5)]:

$$
V_{\text {in }}=-V_{b}\left(1+2 b+\frac{d_{0}}{W}\right) \simeq V_{t h}\left(1+\frac{d_{0}}{W}\right) .
$$

The hole charge provides an effective screening of the transverse electric field in the gated section. This leads to weakening of the sensitivity of the barrier height and the source-drain current on the top gate voltage $V_{t}$ [15] and, hence, to a decrease in the transconductance. This pattern is valid at the dc voltages or when the characteristic time of their variation is long in comparison with the characteristic times of the thermogeneration of holes and the tunneling between the channel side regions and the gated section [15, 17]. In the situation when the hole recharging of the gated section of the channel is a relatively slow process, the ac transconductance at the frequencies higher than the characteristic recharging frequency can substantially exceed the dc transconductance.

\section{Interband tunneling}

At elevated top-gate voltages $\left(V_{t}<V_{i n}\right)$, the interband tunneling of electrons from the conduction band in the source to the valence band in the gated section as well as from the valence band in the gated section to the conduction band in the drain can be essential. At high drain voltages the latter tunneling processes can be particularly pronounced. This can result in elevated source-drain current despite rather high barrier. To limit the tunneling, the back-gate voltage, which mainly determines the energy gap, should be high enough. A decrease in the gate layer thicknesses also promotes the tunneling suppression. For example, if $W=(5-10) \mathrm{nm}$, $V_{b}=-V_{t}=5 \mathrm{~V}$, the energy gap in the gated section of the channel $E_{g} \simeq 0.17-0.34 \mathrm{eV}$ [see Eq. (A4)]. Despite some attempts to calculate the tunneling currents in GFETs and GBL-FETs (see, for instance, Refs. 11, 19]), the problem for GBL-FETs remains open. This is because the spatial nonuniformity of the energy gap in the channel and its nonlinear spatial dependence (particularly near the drain edge) associated with the features of the potential distribution under the applied voltages. Generalizing Eq. (A4), we obtain $E_{g}=e d\left[V_{b}-\varphi(x)\right] / 2 W$, hence the energy gap varies from $E_{g}=E_{g, s} \simeq e d V_{b} / W$ at $x=-L_{t} / 2$ to $E_{g}=E_{g, s} \simeq e d\left(V_{b}-V_{d} / 2 W\right.$ at $x=L_{t} / 2$. It reaches a maximum $E_{g}=d\left(e V_{b}+\Delta_{m}\right) / 2 W$ at $x=x_{m}$. One can see that $E_{g, d}$ can be markedly smaller than $E_{g}$, especially at not too small drain voltages. Due to this, the deliberation of the tunneling in GBL-FETs requires sufficiently rigorous device model (which could include the formulas for the potential distribution obtained above) and numerical approach.

\section{CONCLUSIONS}

We demonstrated that the developed device model of allows to derive the GBL-FET characteristics: the potential distributions along the channel and the dependences of the source-drain current and the transconductance on the applied voltages and the geometrical parameters as closed-form analytical expressions. The key element of the model, which provide an opportunity to solve the problem analytically, is the use of the Poisson equation in the weak nonlocality approximation. In particular, the model accounts for the effect of screening of the transverse electric field by the electron charge in the channel, the short-gate effect, and the effect of drain-induced barrier lowering. The parameters $\eta_{0}$ and $\eta$ characterizing the strength of these effects in the cases of essentially depleted channel and strong screening were expressed via the geometrical parameters and the Bohr radius. As shown, the GBL-FET transconductance exhibits a pronounced maximum as a function of the topgate voltage swing. The interplay of the short-gate effect and the electron collisions results in a nonmonotonic dependence of the transconductance on the topgate length. The obtained analytical formulas for the potential barrier height, the source-drain current, and the transconductance can be used for GBL-FET optimization by proper choice of the thicknesses of gate layers, 
the top-gate length, and the bias voltages.

\section{Acknowledgments}

The authors are grateful to H. Watanabe for stimulating comments and to V. V. V'yurkov for providing Ref. [31]. The work was supported by the Japan Science and Technology Agency, CREST, Japan.

\section{Appendix}

Disregarding the effect of "Mexican-hat" and the nonparabolicity of the electron energy spectrum, the density of states can be considered independent of the energy (in the energy range under consideration). Taking this into account, the electron Fermi energies and the energy gaps in the source and drain sections of the GBL-FET channel are, respectively, given by [17, 18] $\left(W_{b}=W_{t}=W\right)$

$$
\begin{gathered}
\varepsilon_{F, s}=\frac{k_{B} T}{(1+b)} \ln \left[\exp \left(\frac{b e V_{b}}{k_{B} T}\right)-1\right], \\
\varepsilon_{F, d}=\frac{k_{B} T}{(1+b)} \ln \left[\exp \left(\frac{b e\left(V_{b}-V_{d}\right)}{k_{B} T}\right)-1\right], \\
E_{g, s}=\frac{e d V_{b}}{2 W}, \quad E_{g, s}=\frac{e d\left(V_{b}-V_{d}\right)}{2 W} .
\end{gathered}
$$

Here $a_{B}=k \hbar^{2} / m e^{2}, b=a_{B} / 8 W$, and $d \lesssim d_{0}$, where $d_{0} \simeq 0.34 \mathrm{~nm}[30]$ is the spacing between the graphene layers in the GBL, while $d$ stands for the effective spacing accounting for the screening of the transverse electric field by GBL (polarization effect). In portion of the gated section essentially occupied by electrons and its depleted portion, one obtains

$$
E_{g}=\frac{e d\left(V_{b}-V_{t}\right)}{2 W}, \quad E_{g}=\frac{e d_{0}\left(V_{b}-V_{t}\right)}{2 W}
$$

respectively. Due to $a_{B} \gg d$, from Eqs. (A1) - (A4) one obtains $\varepsilon_{F, s} \geq \varepsilon_{F, d}>E_{g, s} \geq E_{g, d}$. At $V_{t}<0, E_{g}$ can significantly exceed $E_{g, s}$ and $E_{g, d}$. In the case of strong degeneracy of the electron system, Eqs. (A1) and (A2) yield

$$
\varepsilon_{F, s} \simeq e V_{b} \frac{a_{B} / 8 W}{(1+b)}, \quad \varepsilon_{F, d} \simeq e\left(V_{b}-V_{d}\right) \frac{b}{(1+b)}
$$

The quantity $\varepsilon_{F, d}$ is given by the same equations in which, however, $V_{b}$ is substituted by $V_{b}-V_{d}$. As a result,

$$
\begin{gathered}
\varepsilon_{F, s}-\varepsilon_{F, d} \simeq \frac{b e V_{d}}{1+b} \simeq b e V_{d}, \\
e V_{d}^{*}=e V_{d}+\varepsilon_{F, d}-\varepsilon_{F, s} \simeq \frac{e V_{d}}{1+b} .
\end{gathered}
$$

Since parameter $b$ in reality is small, so that $\varepsilon_{F, s}-\varepsilon_{F, d} \simeq$ be $V_{d} \ll e V_{d}$ and $V_{d}^{*} \simeq V_{d}$ we put $\varepsilon_{F, s}=\varepsilon_{F, d}=\varepsilon_{F}$ and substitute $V_{d}^{*}$ by $V_{d}$.
[1] C. Berger, Z. Song, T. Li, X. Li, A. Y. Ogbazhi,R. Feng, Z. Dai, A. N. Marchenkov, E. H. Conrad, P. N. First, and W. A. de Heer, J. Phys. Chem. 108, 19912 (2004).

[2] K. S. Novoselov, A. K. Geim, S. V. Morozov, D. Jiang, M. I. Katsnelson, I. V. Grigorieva, S. V. Dubonos, and A. A. Firsov, Nature 438, 197 (2005).

[3] A. H. Castro Neto, F. Guinea, N. M. R. Peres, K. S. Novoselov, and A. K. Geim, Rev. Mod. Phys. 81, (2009).

[4] J. Bai, X. Zhong, S. Jiang, Y. Huang, and X. Duan, Nature Nanotechnology 5, 190 (2010).

[5] V. Ryzhii, M. Ryzhii, and T. Otsuji, J. Appl. Phys. 101, 083114 (2007).

[6] F. Rana, IEEE Tran. Nanotechnol. 7, 91 (2008).

[7] F. Xia, T. Murleer, Y.-M. Lin, A. Valdes-Garsia, and P. Avouris, Nat. Nanotechnol. 4, 839 (2009).

[8] V. Ryzhii, M. Ryzhii, V. Mitin, and T. Otsuji, J. Appl. Phys. 107, 054512 (2010).

[9] V. Ryzhii, A. A. Dubinov, T. Otsuji, V. Mitin, and M. S. Shur, J. Appl. Phys. 107, 054505 (2010).

[10] V. Ryzhii, M. Ryzhii, and T. Otsuji, Appl. Phys. Express 1, 013001 (2008).

[11] V. Ryzhii, M. Ryzhii, and T. Otsuji, Phys. Status Solidi (a) 205, 1527 (2008).

[12] Y. Quyang, Y. Yoon, J. K. Fodor, and J. Guo, Appl. Phys. Lett. 89203107 (2006).

[13] G. Fiore and G. Iannaccone, IEEE Electron Device Lett. 28, 760 (2007).

[14] G. Liang, N. Neophytou, D. E. Nikonov, and M. S. Lundstrom, IEEE Trans. Electron Devices 54, 677 (2007).

[15] V. Ryzhii, M. Ryzhii, A. Satou, and T. Otsuji, J. Appl. Phys. 103, 094510 (2008).

[16] M. Ryzhii, A. Satou, V. Ryzhii, and T. Otsuji, J. Appl. Phys. 104, 114505 (2008).

[17] V. Ryzhii, M. Ryzhii, A. Satou, T. Otsuji, and N. Kirova, J. Appl. Phys. 105, 104510 (2009).

[18] M. Ryzhii and V. Ryzhii, Phys. Rev. B 79, 245311 (2009).

[19] M. Cheli, G. Fiori, and G. Iannaccone, IEEE Trans. Electron Devices 56, 2979 (2009).

[20] S. O. Koswatta, S. Hasan, M. S. Lundstrom, M. P. Anantram, and D. E. Nikonov, Appl. Phys. Lett. 89, 023125 (2006).

[21] M. Lenzi, P. Palestri, E. Gnani, S. Reggiani, A. Gnudi, D. Esseni, L. Selmi, and G. Baccarani, Trans. Electron Devices 55, 2087 (2008).

[22] S. Fregonese, J. Gouet, C. Manex, and T. Zimmer, IEEE 
Trans. Electron Devices 56, 1184 (2009).

[23] K. Natori, J.Appl. Phys. 76, 4881 (1994).

[24] A. Rahman, J. Guo, S. Datta, and M. S. Lundstrom, Trans. Electron Devices 50, 1853 (2003).

[25] F. G. Pikus and K. K. Likharev, Appl. Phys. Lett. 71, 3661 (1997).

[26] V. A. Sverdlov,T. J Walls, and K. K. Likharev, Trans. Electron Devices 50, 1926 (2003).

[27] N. Sano, Phys. Rev. Lett. 93, 246803 (2004).

[28] G. Mugnaini and G. Iannaccone, Trans. Electron Devices 52, 1802 (2005).

[29] R. Kim, P. A. Neophytou, G. Klimeck, and M. S. Lundstrom, J. Vac. Sci. Technol. B26, 1628 (2008).

[30] R. Akis, N. Faralli, D. K. Ferry, S. M. Goodnick, K. A. Phatak, and M. Saratini, IEEE Trans. Electron Devices 56, 2935 (2009).

[31] A. N. Khomyakov and V. V. V'yurkov, Russian Micro- electronics, 38, 393 (2009).

[32] T. Ohta, A. Q. Bostwick, T. Seyller, K. Horn, and E. Rotenberg, Science 333, 951 (2006).

[33] E. McCann, Phys. Rev. B 74, 161403 (2006).

[34] E. V. Castro, K. S. Novoselov, S. V. Morozov, N. M. R. Peres, J. M. B. Lopes dos Santos, L. Nilsson, F. Guinea, A. K. Geim, and A. H. Castro Neto, J. Phys.: Condens. Matter 22, 175503 (2010).

[35] A. A. Sukhanov and Y. Y. Tkach, Sov. Phys. Semicond 18797 (1984).

[36] A. O. Govorov, V. M. Kowalev, and A. V. Chaplik, JETP Lett. 70, 488 (1999).

[37] S. M. Sze, Physics of Semiconductor Devices (Wiley, New York, 1981).

[38] M. Shur, Physics of Semiconductor Devices (PrenticeHall, New Jersey, 1990). 